\title{
BIOMECHANICAL ANALYSIS OF THE DEADLIFT DURING THE 1999 SPECIAL OLYMPICS WORLD GAMES
}

\author{
Rafael F. Escamilla, Tracy M. Lowry, Daryl C. Osbahr, and Kevin P. Speer \\ Duke University Medical Center, Durham, NC, USA
}

\begin{abstract}
Sumo and conventional deadlifts and high and low skilled lifters were compared during the 1999 Special Olympics World Games. Two video cameras collected $60 \mathrm{~Hz}$ data from 40 subjects, and parameters were quantified at barbell liftoff (LO) and barbell knee passing (KP). The sumo group had a more vertical trunk and horizontal thigh at LO, a less vertical shank at $\mathrm{KP}$, and greater forefoot abduction. The sumo group generated ankle dorsiflexor, knee extensor, and hip extensor moments, while the conventional group produced ankle plantar flexor, knee flexor \& extensor, and hip extensor moments. High skilled lifters had a $40 \%$ greater barbell load, greater knee flexion at LO and greater knee extension at KP, $15 \%$ less vertical bar distance, smaller plantar flexor and hip extensor moment arms at LO and KP, and greater knee extensor moment arms at LO.
\end{abstract}

KEY WORDS: powerlifting, moments, angles, kinematics, kinetics, work

INTRODUCTION: The deadlift, one of three lifts in powerlifting competition, is performed using either a conventional style (narrow stance with arms outside knees) or sumo style (wide stance with arms inside knees). Three studies compared biomechanical parameters between sumo and conventional deadlifts (Cholewicki, McGill, \& Norman, 1991; McGuigan \& Wilson 1996; Escamilla, Francisco, Fleisig, et al., 2000). Escamilla et al. (2000) conducted the only known three-dimensional (3-D) biomechanical analysis of the deadlift. These authors reported several significant kinematic and kinetic differences between sumo and conventional deadlifts during a national powerlifting competition. They also reported that a two-dimensional (2-D) was adequate when analyzing biomechanical parameters during the conventional deadlift, but a 3-D analysis was needed to accurately calculate these parameters during the sumo deadlift. McGuigan \& Wilson (1996) and Cholewicki et al. (1991) also reported kinematic and kinetic differences between sumo and conventional deadlifts during regional and national powerlifting competitions. However, no study has yet examined biomechanical parameters during the deadlift for athletes with mental retardation. Participants in Special Olympics competition have varying levels of mental retardation. Although powerlifters in Special Olympics have coaches, most of these coaches are not experts on proper techniques of performing the deadlift, but rather volunteers. Data from this study will aid coaches and volunteers in teaching proper lifting mechanics to Special Olympics powerlifters, minimize their risk of injury, and enhance performance. It was the purpose of this study to quantify joint and segment angles and ankle, knee, and hip moments and moment arms between sumo and conventional deadlifts during Special Olympics competition. In addition, high and low skilled lifters will be compared to each other for both sumo and conventional deadlifts. High and low skilled lifters performing the deadlift have previously been analyzed by Brown \& Abani (1985) for powerlifters without mental retardation. These authors reported several significant biomechanical differences between low and high skilled lifters. It was hypothesized that the high skilled group would maintain a more erect trunk and generate a smaller hip extensor moment and vertical bar distance compared to the low skilled group.

METHODS: Forty male powerlifters, 20 performing the conventional deadlift and 20 performing the sumo deadlift, served as subjects. Mean age, body mass, body height, and load lifted were $25 \pm 6 \mathrm{y}, 76 \pm 19 \mathrm{~kg}, 168 \pm 8 \mathrm{~cm}$, and $126 \pm 32 \mathrm{~kg}$, respectively, for the sumo group, and $25 \pm 4 \mathrm{y}$, $76 \pm 13 \mathrm{~kg}, 167 \pm 10 \mathrm{~cm}$, and $138 \pm 49 \mathrm{~kg}$, respectively, for the conventional group. The Schwartz score, which is used in powerlifting competition to normalize the barbell load to each lifter's body 
mass, was employed to rank from high to low the 20 subjects in the conventional group and the 20 subjects in the sumo group. The top 10 Schwartz score rankings in the sumo and conventional deadlift groups were classified as high skilled lifters, while the bottom 10 Schwartz score rankings in the sumo and conventional deadlift groups were classified as low skilled lifters. Two synchronized gen-locked video cameras were used to collect $60 \mathrm{~Hz}$ video data. A $2 \times 1.5 \times$ $1 \mathrm{~m}$ 3-D calibration frame, surveyed with a measurement tolerance of $0.5 \mathrm{~cm}$, was positioned and videotaped in the same volume occupied by the lifter-barbell system. A 3-D video system was used to manually digitize data for all 40 subjects. A 15 point spatial model was created, comprised of the top of the head and centers of the left and right mid-toes, ankles, knees, hips, shoulders, hands, and end of bar. All points were seen in each camera view. Each of these 15 points was digitized in every video field $(60 \mathrm{~Hz})$. A fourth-order, zero lag Butterworth digital filter was used to smooth the raw data with a cutoff frequency of $5 \mathrm{~Hz}$ (Escamilla et al., 2000). Using the direct linear transformation, 3-D coordinate data were derived from the 2-D digitized images from each camera view. An average resultant mean square calibration error of $0.3 \mathrm{~cm}$ produced an average volume percent error of 0.121 . Three events were defined during the deadlift. The first was barbell liftoff (LO), which was the first picture in which the barbell discs on both sides of the bar were no longer in contact with the lifting platform. The next event was at the instant the bar passed the knees (KP). The last event was lift completion (LC), which occurred when the lifter was in an upright position with the knees and hips fully extended and the shoulders thrust back. To calculate joint and segment angles and hip moments and moment arms, a 3-D orthogonal axis system was translated to the right ankle joint and rotated so that the positive $X$ axis pointed to the left ankle joint, the positive Z-axis pointed anteriorly, and the $Y$-axis pointed vertically (Escamilla et al., 2000). Hip moments were calculated about the X-axis and hip moment arms were calculated in the Z-axis direction. The axes system was then translated to each ankle joint center and rotated so that the positive Z-axis pointed in the direction of the midtoes, the $Y$ axis pointed vertical, and the $X$-axis was orthogonal to the $Y$ and $Z$ axes. Subsequently, ankle and knee moments were calculated about the $X$-axis and ankle and knee moment arms were calculated in the Z-axis direction. Linear and angular displacements were calculated for both the left and right sides of the body, and then averaged (Escamilla et al., 2000). Relative knee and hip angles and absolute trunk, thigh, and shank angles were defined in accordance with previous studies (Brown \& Abani 1985; McGuigan \& Wilson 1996; Escamilla et al., 2000). Trunk, thigh, and shank angles were measured relative to the $X-Z$ horizontal plane. Knee angles were measured relative to the thigh and leg segments, while hip angle was defined as the relative angle between the trunk (hip to shoulder segment) and thigh (hip to knee segment). Foot angle was defined as the angle formed between the foot segment and the $Y-Z$ sagittal plane. Stance width was defined as the linear distance between the left and right ankle centers, while hand width was defined as the linear distance between the left and right hand centers. Left and right hip, knee, and ankle moments and moment arms were calculated at LO, $\mathrm{KP}$, and LC, and then averaged (Escamilla et al., 2000). Joint moments and moment arms were calculated relative to the barbell center of mass ( $\left.\mathrm{COM}_{\text {bar }}\right)$ (Escamilla et al., 2000). Ankle moment arms $\left(\mathrm{MA}_{\text {ankle }}\right)$ were calculated as the distance in the Z-axis direction from the ankle joints to $\mathrm{COM}_{\text {bar. }}$. Ankle moments were the product of $\mathrm{MA}_{\text {ankle }}$ and barbell weight. Knee moment arms $\left(\mathrm{MA}_{\mathrm{knee}}\right)$ were calculated as the distance in the Z-axis direction from the knee joints to $\mathrm{COM}_{\mathrm{bar}}$. Knee moments were the product of $M_{\text {knee }}$ and barbell weight. Hip moment arms $\left(M A_{\text {hip }}\right)$ were calculated as the distance in the Z-axis direction from the hip joints to $\mathrm{COM}_{\text {bar }}$. Hip moments were the product of $\mathrm{MA}_{\text {hip }}$ and barbell weight. Vertical bar displacement was calculated from LO to LC, and normalized by body height. Mechanical work, which was calculated relative to the barbell weight, was the product of the barbell weight and total vertical displacement of $\mathrm{COM}_{\mathrm{bar}}$. A two-factor analysis of variance (ANOVA) was employed to test for main effects of deadlift style (sumo versus conventional) and skill level (high versus low). Post hoc comparisons were made 
using the Tukey test to evaluate the significance between pairwise comparisons. The level of significance used was $P<0.01$.

RESULTS AND DISCUSSION: Significant differences are shown in Table 1.

Table 1 Significant Differences in Biomechanical Comparisons (Mean \pm SD) between Sumo and Conventional Deadlift Groups and between High and Low Skilled Deadlift Groups

\begin{tabular}{|c|c|c|c|c|}
\hline & $\begin{array}{l}\text { Sumo } \\
(n=20)\end{array}$ & $\begin{array}{l}\text { Conventional } \\
\quad(n=20)\end{array}$ & $\begin{array}{l}\text { High Skill } \\
(\mathrm{n}=20)\end{array}$ & $\begin{array}{l}\text { Low Skill } \\
(\mathrm{n}=20)\end{array}$ \\
\hline Stance Width $(\mathrm{cm})$ & $73 \pm 12^{a}$ & $37 \pm 10^{a}$ & $58 \pm 24$ & $52 \pm 19$ \\
\hline Stance Width (\% Shoulder Width) & $194 \pm 30^{a}$ & $93 \pm 26^{a}$ & $152 \pm 67$ & $134 \pm 48$ \\
\hline Hand Width $(\mathrm{cm})$ & $50 \pm 10^{a}$ & $63 \pm 10^{a}$ & $55 \pm 11$ & $58 \pm 13$ \\
\hline Total Vertical Bar Distance $(\% \mathrm{Ht})$ & $28 \pm 4^{a}$ & $30 \pm 2^{a}$ & $27 \pm 3^{b}$ & $31 \pm 3^{b}$ \\
\hline Total Vertical Bar Distance $(\mathrm{cm})$ & $47 \pm 5^{a}$ & $51 \pm 6^{a}$ & $45 \pm 5^{\mathrm{b}}$ & $52 \pm 6^{\mathrm{b}}$ \\
\hline Total Mechanical Work on Bar $(\mathrm{J})$ & $338 \pm 88^{a}$ & $402 \pm 103^{a}$ & $413 \pm 119^{b}$ & $328 \pm 85^{\mathrm{b}}$ \\
\hline Trunk Angle $\left(^{\circ}\right)$ at LO & $16 \pm 6^{\mathrm{a}}$ & $11 \pm 7^{a}$ & $15 \pm 7$ & $12 \pm 10$ \\
\hline Thigh Angle $\left({ }^{\circ}\right)$ at LO & $143 \pm 6^{a}$ & $136 \pm 6^{a}$ & $139 \pm 5$ & $140 \pm 10$ \\
\hline Knee Angle $\left(^{\circ}\right)$ at KP & $158 \pm 10$ & $159 \pm 6$ & $162 \pm 6^{\mathrm{b}}$ & $155 \pm 7^{b}$ \\
\hline Thigh Angle $\left(^{\circ}\right)$ at KP & $120 \pm 6^{a}$ & $111 \pm 7^{\mathrm{a}}$ & $115 \pm 8$ & $116 \pm 8$ \\
\hline Shank Angle $\left(^{\circ}\right)$ at KP & $74 \pm 6^{a}$ & $83 \pm 4^{a}$ & $77 \pm 8$ & $80 \pm 6$ \\
\hline Foot Angle $\left(^{\circ}\right)$ & $25 \pm 6^{a}$ & $15 \pm 6^{a}$ & $20 \pm 8$ & $21 \pm 8$ \\
\hline Ankle Moment Arm $(\mathrm{cm})$ at LO & $-4.3 \pm 5^{\mathrm{a}}$ & $6.1 \pm 3.7^{a}$ & $-0.8 \pm 6.3^{b}$ & $2.6 \pm 7.1^{b}$ \\
\hline Knee Moment Arm (cm) at LO & $-13.2 \pm 8.0^{a}$ & $-3.3 \pm 5.2^{a}$ & $-10.5 \pm 6.9^{b}$ & $-6.0 \pm 7.1^{\mathrm{b}}$ \\
\hline Hip Moment Arm (cm) at LO & $27.9 \pm 6.2$ & $27.9 \pm 5.2$ & $25.9 \pm 3.2^{b}$ & $29.9 \pm 5.9^{b}$ \\
\hline Ankle Moment $(\mathrm{N} \cdot \mathrm{m})$ at LO & $-59 \pm 67^{a}$ & $83 \pm 57^{a}$ & $-2 \pm 104$ & $27 \pm 84$ \\
\hline Knee Moment $(\mathrm{N} \cdot \mathrm{m})$ at LO & $-170 \pm 125^{a}$ & $-55 \pm 78^{a}$ & $-157 \pm 108^{b}$ & $-68 \pm 114^{b}$ \\
\hline Ankle Moment Arm (cm) at KP & $-5.2 \pm 5.3^{\mathrm{a}}$ & $2.9 \pm 4.0^{\mathrm{a}}$ & $-2.5 \pm 6.0$ & $0.3 \pm 6.2$ \\
\hline Knee Moment Arm (cm) at KP & $-4.8 \pm 5.4^{a}$ & $3.2 \pm 3.2^{\mathrm{a}}$ & $-1.6 \pm 6.1$ & $0.0 \pm 5.9$ \\
\hline Hip Moment Arm (cm) at KP & $23.5 \pm 5.4$ & $21.1 \pm 3.6$ & $20.9 \pm 3.8^{b}$ & $23.8 \pm 4.2^{b}$ \\
\hline Ankle Moment $(\mathrm{N} \cdot \mathrm{m})$ at $\mathrm{KP}$ & $-68 \pm 74^{a}$ & $42 \pm 60^{a}$ & $-31 \pm 92$ & $4 \pm 80$ \\
\hline Knee Moment $(\mathrm{N} \cdot \mathrm{m})$ at $\mathrm{KP}$ & $-66 \pm 80^{a}$ & $45 \pm 49^{a}$ & $-21 \pm 96$ & $-1 \pm 76$ \\
\hline
\end{tabular}

Note: Positive moment arms are anterior to joint; negative moment arms are anterior to joint

aSignificant differences $(P<0.01)$ between sumo and conventional groups

bSignificant differences $(P<0.01)$ between high and low skilled groups

Stance and hand widths were 5-15\% wider compared to the lifters from Escamilla et al. (2000). Since the arms are positioned outside the legs during the conventional deadlift, a wider stance may force a wider hand width. If the stance widens excessively, the arms are forced to deviate from a vertical position, causing the lifter to have to bend over to a greater extent to grip the bar. The wider stance and hand widths may have resulted in the lifters in the current study having 20$25 \%$ greater vertical bar distance from LO to LC compared to lifters in Escamilla et al. (2000). Optimal stance and hand widths will also allow the trunk to be more vertical position at LO. Wider stance and hand widths may have compromised good lifting mechanics at LO, since the trunk positions measured for the sumo and conventional groups (Table 1) is 10-15 less than previously reported (Brown \& Abani 1985; McGuigan \& Wilson 1996; Escamilla et al., 2000). The increased forward trunk tilt at LO resulted in a $10-20^{\circ}$ decrease in hip angles compared to several other studies (Brown \& Abani 1985; McGuigan \& Wilson 1996; Escamilla et al., 2000). 
The increased forward trunk tilt at LO may predispose the spine and back musculature to an increased risk of injury (Cholewicki et al., 1991). Cholewicki et al. (1991) reported that a more upright trunk at LO resulted in less anterior shear force at the lumbar L4/L5 joint. This is especially true in the conventional group, which had significantly greater forward trunk tilt than the sumo group at LO, since there is approximately $10 \%$ greater shear force and moment generated at the L4/L5 joint in the conventional deadlift compared to the sumo deadlift (Cholewicki et al., 1991). Compared to the sumo deadlift in the current study, at LO Escamilla et al. (2000) reported $25-30 \%$ smaller hip moment arms, 30-35\% greater knee moment arms, and three times greater ankle moment arms. This implies the national lifters from Escamilla et al. (2000) used their hip extensors less and their knee extensors and ankle dorsiflexors more compared to the Special Olympic lifters. Hip, knee, and ankle moment arm differences occurred because the national lifters maintained the barbell mass closer to the body compared to the Special Olympics lifters. As the COM of the barbell load moves closer to the body in the sumo deadlift, hip moment arms and moments decrease and ankle and knee moment arms and moments increase. In the sumo deadlift, the greater the feet turn out, the closer the barbell can be positioned to the body. The national lifters maintained the barbell closer to the body partially because they had $17^{\circ}$ greater forefoot abduction compared to the Special Olympics lifters. This implies a forefoot abduction of $40-45^{\circ}$ may be optimal in the sumo deadlift in minimizing hip moment arms and moments. The smaller hip moment arms and moments that result by keeping the barbell mass closer to the body also result in smaller L4/L5 joint moments and shear forces (Cholewicki et al., 1991). This implies that the Special Olympics lifters may increase their risk of injury to the low back by keeping the barbell mass further away from the body. In addition, performance may also be compromised, since increasing hip and L4/L5 moments may also result in less weight being able to be lifted. The greater vertical bar distance for the low skilled group compared to the high skilled group may be one reason why the relative loads lifted by the low skilled groups were less compared to the relative loads lifted by the high skilled loads. This greater vertical bar distance for the low skilled group implies that if the loads lifted were the same for both low and high skilled groups, the low skilled group would have to perform a greater amount of mechanical work on the bar compared to high skilled group. However, since the loads lifted were greater in the high skilled group compared to the low skilled group, the high skilled group performed greater mechanical work. The smaller hip extensor moment arms and larger knee extensor and ankle dorsiflexor moment arms imply that the high skilled group keeps the bar closer to the body compared to the low skilled group. Keeping a weight close to the body during lifting is important in minimizing injury potential, especially to the lower back, since hip and spinal moment arms will decrease. This implies that the low skilled group may have a higher risk of injury compared to the low skilled group. Keeping the weight closer to the body also may enhance lifting performance. In addition, ankle, knee, and hip moment arm data suggest that the high skilled group may employ the hip extensors and ankle plantar flexors to a lesser extent and the knee extensors to a greater extent compared to the low skilled group.

CONCLUSIONS: The sumo deadlift was performed with a more upright trunk and a wider stance compared to the conventional deadlift, which may decrease injury risk in the sumo deadlift and increase injury risk in the conventional group. The sumo deadlift may be more effective in developing the ankle dorsiflexors and knee extensors, while the conventional deadlift may be more effective in developing the ankle plantar flexors and knee flexors. High skilled lifters exhibited better lifting mechanics than low skilled lifters by keeping the bar closer to the body, which may both enhance performance and minimize injury risk. 


\section{REFERENCES:}

Brown, E. W. \& Abani, K. (1985). Kinematics and kinetics of the dead lift in adolescent power lifters. Medicine and Science in Sports and Exercise, 17, 554-66.

Cholewicki, J., McGill, S.M., \& Norman, R.W. (1991). Lumbar spine loads during the lifting of extremely heavy weights. Medicine and Science in Sports and Exercise, 23,1179-86.

Escamilla, R. F., Francisco, A.C., Fleisig, G.S., Barrentine, S.W., Welch, C.M., Kayes, A.V., Speer, K.P., \& Andrews, J.R. (2000). A three-dimensional biomechanical analysis of sumo and conventional style deadlifts. Medicine and Science in Sports and Exercise, 32, 1265-1275. McGuigan, M. R. M., \& Wilson, B.D. (1996). Biomechanical analysis of the deadlift. Journal_of Strength and Conditioning Research, 10, 250-55. 